\title{
METATEORIA, CIÊNCIA DA INFORMAÇÃO E ORGANIZAÇÃO DO CONHECIMENTO: UM ES- TUDO A PARTIR DA LITERATURA CIENTÍFICA DA ÁREA
}

\author{
Metatheory, Information Science, and Knowledge Organization: a study based on scientific literature
}

Juan Bernardo Montoya-Mogollón (1) Rafael Cacciolari Dalessandro (2) Natália Bolfarini Tognoli (3)

(1) Universidade Estadual Paulista, juan.mogollon@marilia.unesp.br (2) rafaelcacciolari@marilia.unesp.br (3) Universidade Federal Fluminense, nataliatognoli@id.uff.br

\begin{abstract}
Resumo
Conhecida como a área do conhecimento que teoriza sobre outras teorias, a metateoria, nos últimos anos, encontrou terreno fértil nos estudos da Ciência da Informação e da Organização do Conhecimento preocupando-se com as filosofias e metodologias empregadas nesse domínio. O presente trabalho tem como objetivo verificar como os estudos metateóricos são apresentados no domínio da Ciência da Informação e da Organização do Conhecimento no âmbito da literatura científica da área. Para tanto, partimos da análise dos trabalhos indexados nas bases de dados Library and Information Science Abstracts (LISA) e SCOPUS que continham em seu título e/ou palavras-chave o termo "metateoria" Os dados foram analisados sob a ótica dos estudos metateóricos de Ritzer e os resultados demonstraram uma pluralidade de abordagens discutidas na Ciência da Informação e na Organização do Conhecimento, o que confirma a aplicabilidade da metateoria no âmbito dos estudos desse domínio, caracterizando-o como um promissor campo de pesquisa nessa temática.
\end{abstract}

Palavras-chave: Teoria; Metateoria; Organização do conhecimento; George Ritzer; Literatura científica

\section{Introdução}

A Ciência da Informação (CI), assim como qualquer outra área do conhecimento, explicita o seu conjunto de saberes através de seus métodos científicos, que se configuram por um conjunto de regras básicas visando o desenvolvimento de uma experiência em um novo conhecimento e/ou correção e integração de conhecimentos já existentes. Para tanto, tais métodos são baseados em observações, hipóteses, leis e teorias.

Para Wallis (2010), a ciência, em uma clara analogia aos pássaros, deve ter duas fortes asas caso queira voar, argumentando que fatos empíricos são entendidos à luz de uma teoria específica e a teoria é construída a partir da consideração cuidadosa dos fatos. Desse modo, teoria e pesquisa são tão entrelaçadas que uma não existe sem a outra, sendo ambas necessárias para uma ciência mais forte.

\begin{abstract}
Metatheory is known as an area of knowledge that theorizes about other theories, which has found in recent years a fertile ground within Knowledge Organization field minding the philosophies and methodologies employed in this domain. The present paper aims to verify how metatheoretical studies are presented in $\mathrm{KO}$ in the scope of its scientific literature. Therefore, the works available in Library and Information Science Abstracts (LISA) and SCOPUS, which had the metatheory word in its title and keywords, were analyzed. The analysis was carried out according to Ritzer's metatheory studies and the results have shown a plurality of approaches discussed within $\mathrm{KO}$, which confirms the applicability of metatheory in the scope of this domain, characterizing itself as a promising field of research in the area.
\end{abstract}

Keywords: Theory; Metatheory; Knowledge Organization; George Ritzer; Scientific Literature.

As ciências sociais possuem como objeto de estudo as atividades e o comportamento dos seres humanos. Por se tratar de uma área interdisciplinar das ciências sociais aplicadas que consiste em analisar, classificar, armazenar, recuperar, disseminar, assim como outros aspectos envolvidos na gestão da informação, a Ciência da Informação engloba várias teorias e métodos no intuito de viabilizar tais processos, caracterizando-se assim um campo fértil para a utilização da metateoria.

A metateoria é tida como área do conhecimento que teoriza sobre teorias de outros campos científicos, normalmente duas ou mais, conferindo-lhes legitimidade, uma vez que se não a seguirmos, as teorias ficam desacreditadas (Laze, 2013). A metateoria preocupa-se, portanto, com a investigação, análise e descrição das teorias.

A importância deste trabalho centra-se em refletir como a metateoria pode ser um campo epistemológico que fornece um profícuo conhecimento para ser aplica-

Montoya-Mogollón, Juan Bernardo; Dalessandro, Rafael Cacciolari; Tognoli, Natália Bolfarini. Metateoria, Ciência da Informação e Organização do Conhecimento: um estudo a partir da literatura científica da área. // Brazilian Journal of Information Studies: Research Trends. 12:2 (2018) p.6-12. 
do nas áreas da Ciência da Informação e da Organização do conhecimento, já que reflete na própria ciência, problematizando suas estruturas.

Inúmeros são os trabalhos que atentam para uma conceituação indefinida da metateoria, resultando em uma série de trabalhos isolados, subáreas isoladas com tipos de metateorização e isolados tipos de metateorização, dificultando ainda mais a compreensão da temática.

Um campo fértil nos estudos da Ciência da Informação para a metateoria é a Organização do Conhecimento (OC), onde os trabalhos de Vickery (1997), Svenonius (2004), Bates (2005) e Tennis (2008) se destacam.

A Organização do Conhecimento é definida por Hjorland (2008) como um campo de pesquisa, ensino e prática cujo objetivo é descrever, representar, preencher e organizar documentos e representações documentais assim como assuntos e conceitos, tanto por pessoas, quanto por programas de computador. Nesse contexto, a Organização do Conhecimento juntamente com seus processos (catalogação, análise de assunto, indexação e classificação) e sistemas (sistemas de classificação, lista de cabeçalhos de assunto, tesauros, ontologias e sistemas de metadados), podem ser considerados fundamentais no contexto da Ciência da Informação, Arquivologia e Biblioteconomia, uma vez que a organização pressupõe qualquer tipo de acesso e apropriação de conhecimento no âmbito dos serviços de informação ligados a essas disciplinas.

Segundo Bates (2005) nas ciências sociais é mais comum - ao contrário das ciências naturais - ter um paradigma geral para um campo que descreva o domínio de interesse para aquela disciplina, enquanto lida com mais de uma metateoria, ou filosofia de pesquisa à qual o pesquisador se identifica. Nesse contexto, é possível que um pesquisador esteja associado a mais de uma metateoria, uma vez que podemos compreendê-la como uma filosofia por trás da teoria, preocupada em compreender um fenômeno de interesse em um domínio específico.

A autora define teoria como sendo todo corpo de conhecimento de generalizações e princípios desenvolvidos por um campo, um sistema de pressupostos, princípios e relações colocadas para explicar um conjunto especifico de fenômenos, a exemplo da CI (BATES, 2005). Normalmente, as teorias contêm uma metateoria, metodologia e regras de procedimentos implícitas.

Para Tennis (2008), as teorias são um conjunto de proposições usadas para explicar um fenômeno, narrativas. Enquanto narrativas, algumas podem prever ou reformular nossa visão de mundo, assim como outras são criadas para mudar nossa visão referente às ações sociais. Desse modo, a metateoria tem como intenção providenciar narrativas sobre essas teorias.

Dentre os autores relevantes sobre o tema destaca-se o trabalho de Ritzer (1991) (que, embora aplique a me- tateoria no âmbito da Sociologia, tem seus trabalhos utilizados por autores da Ciência da Informação e da Organização do Conhecimento), em que o mesmo propõe a existência de três variantes metateóricas, segundo o objetivo: (1) fornecer uma compreensão mais profunda do trabalho científico existente $(\mathrm{Mu})$ ou seja, estudar uma teoria a fim de aprofundá-la e conhecê-la melhor; (2) servir como um prelúdio para um trabalho teórico futuro (Mp) utilizando, assim, uma teoria existente para criar ou desenvolver uma nova teoria; e (3) a metateorização como fonte das perspectivas que sustentam a teoria sociológica: fornecer uma perspectiva abrangente desse trabalho (Mo).

Considerando as diversas metateorias empregadas nos estudos da Ciência da Informação e na Organização do conhecimento (Bates atenta para pelo menos 13 metateorias) e a fim de estabelecermos como os estudos metateóricos se apresentam no domínio da Organização do Conhecimento no âmbito da literatura científica da área, partimos da análise dos trabalhos indexados nas bases de dados Library and Information Science Abstracts (LISA) e SCOPUS a partir das três variantes metateóricas propostas por Ritzer verificando especificamente como a variante $(\mathrm{Mu})$ está estruturada nos textos. Assim, utilizamos o quadrante no qual se encontram as dimensões externa e interna e, intelectual e social dos estudos metateóricos, a fim de identificarmos as metateorias subjacentes nos trabalhos que tratam justamente do tema.

Pretendemos com esse estudo lançar luz aos estudos metateóricos no âmbito da Ciência da Informação e da Organização do Conhecimento, haja vista sua importância para a compreensão da filosofia e da história por trás do desenvolvimento de uma abordagem de pesquisa.

\section{Procedimentos metodológicos}

O corpus da pesquisa foi obtido através de buscas em duas bases de dados internacionais, Library and Information Science Abstracts (LISA) e SCOPUS no dia 01/09/2016, a partir do termo "metatheory" nos campos de título, resumo e palavras-chave. Foram obtidos 10 resultados na LISA e 16 na SCOPUS, no entanto os textos recuperados na SCOPUS eram referentes à recuperação da informação e dois na LISA eram sobre assuntos diversos. Como o objetivo da busca foi obter artigos a respeito da metateoria no contexto da Ciência da Informação e da Organização do Conhecimento, os 18 trabalhos que não tratavam do tema foram excluídos, recaindo-se a análise sobre os 8 textos restantes

A compilação dos textos resultou em um conjunto documental representante de um domínio, ou seja, um determinado campo de especialidade. A partir de então, passamos a analisar esse domínio específico de artigos sobre a relação da metateoria com a Organização do Conhecimento. Como destaca Smiraglia (2011), a aná-

Montoya-Mogollón, Juan Bernardo; Dalessandro, Rafael Cacciolari; Tognoli, Natália Bolfarini. Metateoria, Ciência da Informação e Organização do Conhecimento: um estudo a partir da literatura científica da área. // Brazilian Journal of Information Studies: Research Trends. 12:2 (2018) p.6-12. ISSN 1981-1640. 
lise de domínio caracteriza-se pelo estudo dos aspectos teóricos de um dado entorno, geralmente representado por uma literatura ou comunidade de pesquisadores, constituindo um meio para a geração de novo conhecimento acerca da interação de uma dada comunidade científica com a informação.

Nesse contexto, pretendemos compreender teoricamente como essa literatura selecionada em Organização do Conhecimento trabalha as questões metateóricas, ou seja, qual a posição epistêmica que se destaca nesse âmbito específico.

Para tanto, os textos selecionados foram analisados dentro do esquema proposto por George Ritzer (1991) no qual explicita a existência de três variantes metateóricas anteriormente mencionadas. Dentre essas três, a variante $(\mathrm{Mu})$ foi escolhida para que a literatura fosse analisada, uma vez que esta é responsável por fornecer uma compreensão mais profunda do trabalho científico existente.

A análise a partir da variante Mu é feita com base no quadrante proposto pelo autor a partir das seguintes dimensões: interna e externa, e intelectual e social, podendo um estudo ser: intelectual-interno, socialinterno, intelectual-externo e social-externo.

Cada dimensão possui suas peculiaridades, não sendo, no entanto, rigidamente distintas ou opostas umas das outras, podendo-se ter trabalhos que apresentam as quatro dimensões ao mesmo tempo, por exemplo. Desse modo, os dados contidos nos trabalhos selecionados foram tabelados a partir das quatro dimensões com intuito de compará-los e atingir os objetivos mencionados.

\section{Análise e Discussão dos dados}

Conforme explicitado na seção anterior, os trabalhos selecionados foram analisados a partir das quatro dimensões de $(\mathrm{Mu})$ propostas por Ritzer. Como se observa no quadro 1 , no interior da variante $(\mathrm{Mu})$, existem subcategorias que aprofundam e detalham a teoria da área de estudo: interna/externa e intelectual/social.

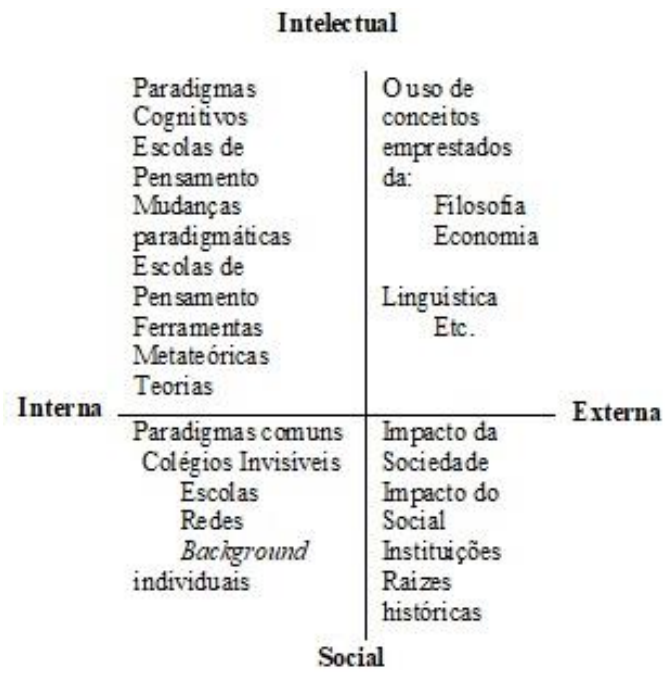

Quadro I. Quadrante de $M u$

A dimensão interna faz referência ao aprofundamento da área, àquilo que lhe é inerente, enquanto a externa se refere aos conceitos que se encontram fora dela, mas que exercem alguma influência sobre a mesma. A dimensão intelectual é entendida como aquilo que se relaciona com a composição cognitiva da área, teorias, ferramentas, ideias de outras disciplinas, enquanto a dimensão social sustenta a composição sociológica da área (Tognoli, 2015).

Na primeira abordagem (Interna-Intelectual), é possível verificar os paradigmas pelos quais passam as ciências, escolas de pensamento ou correntes teóricas unidas a partir das teorias da área estudada, bem como as ferramentas e métodos utilizados para analisar as teorias e os teóricos.

A segunda (Interna-Social) foca os aspectos comuns entre as várias teorias da área, com ênfase em pequenos grupos de teóricos diretamente ligados e suas relações, bem como suas afiliações institucionais.

A terceira (Externa-Intelectual) diz respeito aos conceitos, teorias e elementos emprestados de outras disciplinas, fazendo uso delas para analisar sua teoria.

A quarta abordagem (Externa-Social) está diretamente ligada ao impacto da sociedade na construção da teoria.

Em qualquer abordagem metateórica (vide quadro 1), podem ser encontradas uma, duas, três ou as quatro categorias de $\mathrm{Mu}$, já que as mesmas se complementam e não são excludentes, como destaca Ritzer (1991, p. 17):

[...] antes de proceder devo adicionar algumas notas cautelosas. Primeiro, as duas dimensões (interna-externa; intelectual-social) são uma continuação, sem linhas duras e estáticas entre seus polos. Segundo os quatro tipos de $\mathrm{Mu}$ desenvolvidos ao transpassarem essas linhas não são rigidamente diferentes uns dos outros. Em outras palavras, em

Montoya-Mogollón, Juan Bernardo; Dalessandro, Rafael Cacciolari; Tognoli, Natália Bolfarini. Metateoria, Ciência da Informação e Organização do Conhecimento: um estudo a partir da literatura científica da área. // Brazilian Journal of Information Studies: Research Trends. 12:2 (2018) p.6-12. 
trabalhos metateóricos específicos, podem confluir dois, três, ou quatro tipos de Mu.

Os 8 textos recuperados nas buscas são apresentados a seguir:

1. Lor, Peter Joan. Revitalizing comparative library and information science: theory and metatheory

2. Rioux, Kevin. Metatheory in Library and Information Science: A Nascent Social Justice Approach

3. Talja, Sanna, Touminem, Kimmo, Savolainen Reijo. "Isms" in information science: constructivism, collectivism and constructionism.

4. Vamanu, Lulian. Hermeneutics: a sketch of a metatheoretical framework for library and information science research

5. Bonnevie, Ellen. Dretke's semantic information theory and metatheory in library and information Science

6. Castanha, Renata, Gracio, Maria Claudia. Bibliometrics contribution to the metatheoretical and domain analysis studies.

7. Dow, John. A metatheory for the development of a science of information

8. Hjorland, Birger. Theory and metatheory of information science: a new interpretation

Tabela I. Trabalhos recuperados

No primeiro artigo, "Revitalizing comparative library and information science: theory and metatheory", o autor realiza um estudo comparativo entre a Biblioteconomia e a Ciência da Informação, tendo como referência hipóteses metateóricas em dimensões sociológicas, teleológicas, ontológicas, epistemológicas e éticas.

A análise demonstra destarte que o trabalho de Lor, enquadra-se primeiramente na categoria: intelectual/interna, uma vez que o autor trata de mudanças paradigmáticas, quando emprega a importância do uso de paradigmas como o positivismo, pós-positivismo, teorias críticas e paradigmas relacionados e construtivismo em LIS (Library and Information Science); ferramentas metateóricas, quando o autor estabelece o uso de conceitos sociológicos, teleológicos, epistemológicos e éticos. $\mathrm{O}$ autor explica, também, a emergência do uso de teorias quando apresenta a importância de se aprofundar no uso delas dentro da mesma LIS, já que as teorias existentes "são feitas a partir dos outros campos de estudo como a psicologia, a sociologia ou a gestão" (Lor, 2014).

O autor continua sua argumentação no campo da teoria em seu trabalho, apontando a importância de encontrar explicações teóricas que podem ser aplicadas a mais de um estudo comparativo, procurando empréstimos de outras disciplinas como, por exemplo, as ciências sociais. Ainda segundo o autor, esse tipo de estudo comparativo aprofunda o campo da pesquisa e, no caso da LIS, outras visões nas ciências sociais podem construir teorias sobre a difusão das inovações, mudanças culturais, empréstimos de política, desenvolvimento e póscolonialidade (Lor, 2014).

Outra dimensão encontrada no artigo de Lor é a social/externa, no âmbito do impacto do social, ao ser considerada a atenção no campo do social e cultural, entre outros fatores, para a contribuição da LIS no âmbito internacional (Lor, 2014).

No segundo artigo, "Metatheory in Library and Information Science: a Nascent Social Justice Approach", o autor propõe a justiça social como uma metateoria para a Biblioteconomia e Ciência da Informação, advertindo a necessidade de se criar uma metateoria que discuta e aprofunde a teoria no interior da LIS como mecanismo que possibilite fortalecer a disciplina. A dimensão intelectual/interna é observada quando o autor ressalta o conceito de teoria, destacando que muitas vezes o termo metateoria é usado de forma intercambiável com um ou mais termos como quadro conceitual, modelo, teoria e paradigma (Rioux, 2010). Segundo o autor, isso acontece porque talvez não exista um acordo sobre os conceitos e, portanto, esses dispositivos não ficam tão claros.

A dimensão intelectual/externa é percebida a partir do uso de conceitos emprestados da Filosofia quando o autor inclui elementos metateóricos como um conjunto de hipóteses da natureza da realidade e do ser humano (ontologia), a natureza do conhecimento (epistemologia), os propósitos da teoria e da investigação (teleologia), valores e éticas (axiologia); e a (ideologia) natureza do poder (Rioux, 2010).

No terceiro artigo, "Isms" in information science: constructivism, collectivism and constructionism", os autores realizam uma pesquisa sobre o uso de metateorias como o construtivismo, o coletivismo e o construcionismo nos processos de busca e recuperação de informação, e formação em conhecimento na Ciência da Informação. Essas metateorias, segundo os autores, ampliam a orientação teórica nos estudos da Ciência da Informação para o desenvolvimento de soluções tecnológicas práticas.

Ao analisarmos o trabalho, identificamos a dimensão intelectual/interna no âmbito das seguintes categorias: Paradigmas cognitivos, já que os conceitos que os autores abordam como o construtivismo, são criações individuais de estruturas de conhecimento e modelos mentais, através da experiência e da observação (Talja, Touminen \& Savolainen, 2005); Ferramentas metateóricas, porquanto os autores estabelecem que os conceitos de construtivismo, coletivismo e construcionismo são metateorias divergentes na Ciência da Informação (Talja, Touminen \& Savolainen, 2005); e Teorias, quando os autores explicam que o artigo não tenta criar uma classificação detalhada de pesquisas individuais na $\mathrm{CI}$ ou de teorias dentro das mencionadas posições metateóricas.

Montoya-Mogollón, Juan Bernardo; Dalessandro, Rafael Cacciolari; Tognoli, Natália Bolfarini. Metateoria, Ciência da Informação e Organização do Conhecimento: um estudo a partir da literatura científica da área. // Brazilian Journal of Information Studies: Research Trends. 12:2 (2018) p.6-12. ISSN 1981-1640. 
Outra dimensão identificada no trabalho dos autores é a intelectual/externa, uma vez que são usados conceitos emprestados da psicologia e da filosofia. Os "ismos" desenvolvidos pelos autores são conceitos trabalhados especialmente pela psicologia, na qual, tiveram importância particularmente com o desenvolvimento dos estudos cognitivos. Os autores explicam que esses "ismos" são emprestados muito mais da psicologia e da educação do que, por exemplo, dos estudos das ciências sociais (Talja, Touminen \& Savolainen, 2005).

No quarto artigo, "Hermeneutics: a sketch of a metatheoretical framework for library and information science research", o autor realiza uma discussão na Biblioteconomia e Ciência da Informação, explorando uma concepção de entendimento desenvolvido dentro da tradição da filosofia da hermenêutica de HansGeorg Gadamer e suas características, como um quadro metateórico na CI (Vamanu, 2013). Segundo o autor, o estudo explica algumas de suas implicações metodológicas para a pesquisa em CI (Vamanu, 2013).

Ao analisarmos o trabalho verificamos que o mesmo se encontra na dimensão intelectual/externa, abordando o uso de conceitos emprestados da filosofia. Tal definição é observada centralmente na leitura, quando o autor explica que o trabalho propõe fazer uma exploração profunda da 'filosofia hermenêutica' (ou interpretativismo hermenêutico), como um quadro metateórico para a investigação na Ciência da Informação.

Essa exploração articula a noção de entendimentos e seus vários aspectos, e oferece sugestões a respeito de como esses procedimentos devem ser desenvolvidos (Vamanu, 2013).

O quinto artigo analisado, "Dretke's semantic information theory and metatheory in library and information Science" apresenta a teoria da informação semântica do filósofo Dretske como contribuição para a discussão da metateoria e suas implicações práticas no campo da Ciência da Informação. A contribuição majoritária da teoria consiste no esclarecimento dos conceitos, mas há problemas relativos às suas aplicações práticas, necessitando de mais estudos.

Ao analisarmos o trabalho de Bonnevie (2001), foi possível identificar a dimensão intelectual/externa, relacionando-o à estrutura cognitiva da área: teorias, ferramentas e ideias de outras disciplinas (filosofia) e fenômenos externos que exercem influência sobre a área.

O sexto artigo, Bibliometrics contribution to the metatheoretical and domain analysis studies, centra-se na contribuição da bibliometria para os estudos metateóricos e de análise de domínio, uma vez que as análises epistemológicas, sociológicas e históricas, fornecem um ponto de vista qualitativo mais completo da área. $\mathrm{O}$ estudo contribui para o discurso dos aspectos teóricos de estudos bibliométricos para apresentar considera- ções concernentes à análise de domínio de Hjorland, como a aproximação metateórica de Ritzer.

Ao analisarmos o trabalho dos autore identificamos duas dimensões: social/interna, uma vez que foca nos aspectos comuns de várias teorias da área, com ênfase em pequenos grupos teóricos ligados e suas relações; e intelectual/externa onde se observam elementos emprestados de outras disciplinas, fazendo uso delas para melhor compreender a área de estudo.

No sétimo artigo, "A metatheory for the development of a science of information", o autor centra-se no uso da metateoria como um guia para o desenvolvimento de melhores teorias na área da CI. Nesse contexto, fica claro que a interdisciplinaridade da Ciência da Informação fundamenta o uso da metateoria para desenvolver essas teorias.

A análise do artigo nos permitiu identificar a dimensão intelectual/externa, uma vez que o autor desenvolve conceitos emprestados de outras disciplinas que exercem influência sobre a CI.

$\mathrm{O}$ oitavo e último artigo, "Theory and metatheory of information science: a new interpretation", discute as hipóteses teóricas e epistemológicas na Ciência da Informação. No trabalho, o autor explica que essas teorias são fundamentais na recuperação da informação, na visão dos documentos e seu papel na comunicação, seleção da informação, nas teorias acerca de funções dos sistemas de informação e no papel do professional da informação (Hjorland, 1998).

A análise do artigo de Hjorland nos permitiu enquadrálo nas dimensões intelectual/interna e intelectual/externa. Na primeira, o autor estabelece as teorias que podem ser usadas na Ciência da Informação, e na segunda, o autor toma conceitos desenvolvidos da Filosofia para compreender melhor a área de estudo.

Os resultados demonstram que das quatro dimensões propostas por Ritzer, na abordagem $\mathrm{Mu}$, a mais recorrente nos trabalhos que abordam a metateoria e sua relação com a Organização do Conhecimento é a intelectual/externa (com sete incidências), sobressaindo o uso de conceitos emprestados de outras disciplinas para a área, neste caso com predominância da Filosofia, reforçando o caráter interdisciplinar da Ciência da Informação e da Organização do Conhecimento.

A segunda abordagem mais recorrente nos trabalhos é a intelectual/interna (com cinco incidências), sendo assim possível observar os paradigmas pelos quais passam as ciências, escolas de pensamento e correntes teóricas, mais impulsionados pelas teorias.

As abordagens social/externa e social /interna foram menos recorrentes nos trabalhos (cada uma com apenas uma incidência), demonstrando que o impacto da sociedade na construção da teoria não é tão discutido no âmbito analisado. 
Como mencionado anteriormente, estas quatro abordagens podem acontecer simultaneamente em um mesmo trabalho, do mesmo modo que duas ou três ao mesmo tempo, como é o caso das abordagens intelectual/interna e intelectual/externa, que são os únicos exemplos de interação aparecendo juntas em quatro trabalhos.

\section{Conclusão}

A metateoria, enquanto uma filosofia por trás da teoria apresenta-se como uma abordagem de pesquisa utilizada para compreender um domínio.

No âmbito da LIS, as metateorias apresentam um duplo papel, ora como filosofia, ora como metodologia. Segundo Bates (2005) no campo filosófico as abordagens metateóricas mais recorrentes são as construtivistas a teoria crítica, a sócio-cognitiva, a cognitiva, entre outras que são mais direcionadas por orientações teóricas e filosóficas, com implicações metodológicas. Já no campo metodológico, podemos encontrar as abordagens bibliométricas, históricas, analítico-filosóficas, entre outras, com algum direcionamento filosófico.

A metateoria é, portanto, uma importante ferramenta que auxilia na delimitação de um domínio, uma vez que é responsável por fornecer fundamentos filosóficos e metodológicos imprescindíveis para um melhor entendimento de um dado campo do conhecimento, como foi possível constatar a partir das quatro dimensões propostas por Ritzer (intelectual/interna; social/interna; intelectual/externa; social/externa) em sua percepção de metateoria enquanto $(\mathrm{Mu})$.

No entanto, sabe-se pouco sobre o domínio da metateoria, o que é notado pela pouca produção científica disponível sobre seu conteúdo e a conseguinte falta de diálogo entre os pesquisadores na tentativa de melhor compreender seus conceitos. Daí a necessidade de caracterizarmos como se dá a literatura científica da área disponível nas bases de dados internacionais LISA e SCOPUS, para melhor assimilar a metateoria a partir destas quatro dimensões.

Constatamos que as abordagens mais comuns são as que utilizam conceitos emprestados de outras disciplinas, a exemplo da Filosofia, representadas pela abordagem intelectual/externa com sete incidências, o que reforça o caráter interdisciplinar da Ciência da Informação e da Organização do Conhecimento; seguida pela observação dos paradigmas pelos quais passam as ciências, escolas de pensamento e correntes teóricas representada pela intelectual/interna, o que demonstra também uma preocupação com a delimitação e explicação do domínio, a partir de sua comunidade discursiva e do conjunto de conhecimento especializado que lhe é inerente.

A tímida incidência das dimensões social/externa e social/interna nos textos analisados chama a atenção para a carência de aspectos que dizem respeito ao impacto da sociedade na construção das teorias e aspectos comuns entre as várias teorias da área na literatura científica analisada.

Os resultados demonstram uma pluralidade de abordagens discutidas no âmbito da Organização do Conhecimento, o que confirma a aplicabilidade da metateoria no âmbito dos estudos desse domínio. No entanto, apesar da pluralidade de abordagens, o número reduzido de artigos encontrados quando refinamos a busca aos artigos sobre Organização do Conhecimento, demostra que, embora efetivo, o estudo metateórico no campo ainda é tímido, configurando-se como um promissor campo de pesquisa nesse domínio.

\section{Referências}

Bates, M. (2005). An introduction to metatheories, theories, and models. // Fische, K.; Erdelez, S.; Mckechnie, L. (Orgs.). Theories of information behavior. Medford: Information Today, 1-24.

Bonnevie, E. (2001). Dretsket's semantic information theory and metatheories in library and information science. // Journal of Documentation 57:4 (2001).

Castanha, R.C.G.; Gracio, M.C.C. (2014). Bibliometrics contribution to the metatheoretical and domain analysis studies. // Knowledge Organization 41 (2014) 171-174.

Dow, J. T. (1977). A Metatheory for the Development of a Science of Information. // Journal of the American Society for Information science 28:6 (1997).

Hjorland, B. (1998) Theory and metatheory of information science: a new interpretation. // Journal of Documentation 54:5 (1998).

Hjorland, B. (2008). What is Knowledge Organization (KO)? // Knowledge Organization 35: 2/3 (2008) 86-101.

Laze, R. (2013). O que é metateoria?. http://brunolaze.blogspot.com.br/2013/01/o-que-emetateoria.html>. (07/12/2016)

Lor, P. J. (2014). Revitalizing comparative library and information science: theory and metatheory. // Journal of Documentation 70:1 (2014).

Rioux, K. (2010). Metatheory in Library and Information Science: A Nascent Social Justice Approach. // Journal of Education for Library and Information Science 51:1 (2010).

Ritzer, G. (1991). Metatheorizing in Sociology. Lexington: Lexignton Books, 1991.

Svenonius, E. (2004). The epistemological foundations of knowledge representation. // Library Trends 52: 3 (2004) 571-587.

Talja, S.; Tuominen, K.; Savolainen, R. (2005). "Isms" in information science: constructivism, collectivism and constructionism. // Journal of Documentation 61:1 (2005).

Tennis, J.T. (2008). Epistemology, Theory, and Methodology in Knowledge Organization: toward a classification, metatheory, and research framework. Knowledge Organization 35: 2 (2008) $102-122$.

Tognoli, N.B. (2015). Metateoria e Diplomática: a construção de um novo modelo de análise para a identificação de documentos de arquivo. // Scire 21 (2015) 75-81.

Vamanu, I. (2013). Hermeneutics: a sketch of a metatheorical framework for library and information science research. // Information Research 18:3 (2013). 
Vickery, B. (1997). Metatheory and information science. // Journal of Documentation 53:5 (1997) 457-476.

Wallis, S.E. (2010). Toward a science of metatheory. // Integral Review 6:3 (2010) 73-120.

Copyright: (C) 2018- Montoya-Mogollón, Dalessandro e Tognoli. This is an open-access article distributed under the terms of the Creative Commons CC Attribution-ShareAlike (CC BY-SA), which permits use, distribution, and reproduction in any medium, under the identical terms, and provided the original author and source are credited.

Received: 2017-08-16. Accepted: 2017-09-20 